\title{
AYURLOG
}

National Journal of Research in Ayurved Science

http://www.ayurlog.com

Nov- $\left.2020\right|^{\text {Volume } 08^{\text {th }}} \mid$ Issue: $6^{\text {th }}$

ISSN: 2320-7329

\section{A review article of Rasapanchak of Manjishtha (Rubia cordifolia Linn.) according to Ayurveda from Brihatrayi \& Various Nighantu.}

\author{
Snehal Kumar Dabhole*1, Priyanka Dilip Chavan², \\ P. G. Scholars ${ }^{1,2}$, Dravyaguna Vidnyan Dept., \\ College of Ayurved \& Research Centre, Akurdi, Pune, Maharashtra, India \\ *Corresponding Author: snehaldabhole8686@gmail.com
}

\begin{abstract}
:
Manjishtha or Indian Maddar is considered to be one of the best blood purifying herbs. It is mainly used to break down blockages in the blood flow and remove stagnant blood. Its root is extensively used in many skin diseases. Manjishtha is widely used in textile industry as a natural red colouring agent.

This review article presents information about Rasapanchak - Rasa, Veerya, Vipak, Guna and Karma of Manjishtha mentioned by Acharya Charak, Sushrut \& Vaghbhat as well by various Nighantukar.
\end{abstract}

Keywords: Manjishtha, Rubia cordifolia, Indian Maddar.

\section{Introduction:}

Name of Manjishtha in Sanskrit translates to "bright red", alluding to its strong affinity to the blood. An extremely important herb in Ayurveda, Manjishtha is a medicinal important plant species of the family Rubiaceae. It is a well-known for its valuable active principals found in it which are used in drug preparation. The ancient Indian Acharya Charak, classified Manjishtha as a "rejuvenative" that acts as a detoxifier. Acharya Sushrut another sage and surgeon recommended it for balancing the bio-elements or life energies of the body for good health.

\section{Materials \& Methodology:}

A detail study of Manjishtha is compiled from all Brihatrayi and various Nighantu.

\section{Vedic Period}

There is no specific reference of the drug Manjistha found in vedic period.

\section{Samhita Period}

The drug Manjishtha mentioned very commonly in Brihatrayi and Laghutrayi. Manjishtha is considered as important drug in the management of Raktapitta, Vranaropan, Shonitaarsha, Raktaatisar etc. It is also used as Varnya in various Twak roga.

\section{Nighantu Period}


As Manjishtha is a commonly used drug since Samhita kala, almost all nighantus have mentioned of Manjistha with various synonyms, rasa, guna, virya, vipak and ganas.

\section{Nirukti of Manjishtha:}

"मज्जिष्ठा -मञ्जौ इोभने वर्णे इरीरं स्थापयतीति,रक्तरोधकत्वात्;

अथवा-मज्जुत्वे सर्वातिशायिनी तिष्ठतीति "। भावदीपिका३
It provides good colour or appears very beautiful.

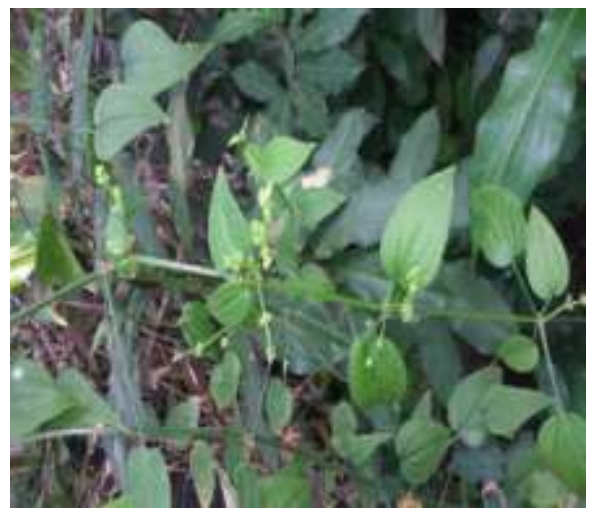

References of Rasapanchak

\begin{tabular}{|c|c|c|c|c|c|c|c|c|c|c|}
\hline \multicolumn{2}{|l|}{ Rasapanchak } & C.S. & S.S. & A.S./ & B.N. & D.N. & M.N. & $K . N$. & $R . N$. & $S g$ \\
\hline \multirow{3}{*}{ Rasa } & Madhur & - & - & - & + & + & + & + & + & + \\
\hline & Tikta & - & - & -8 & + & & + & + & & + \\
\hline & Kashaya & + & - & t & + & + & + & + & + & + \\
\hline \multirow[t]{2}{*}{ Virya } & Ushna & - & -19 & - & + & + & + & + & + & + \\
\hline & Sheet & $p$ & f- & - & - & - & - & - & - & - \\
\hline \multirow[t]{2}{*}{ Vipak } & Madhur & - & - & - & + & + & + & + & + & + \\
\hline & Katu & - & - & - & - & - & - & - & - & - \\
\hline \multirow{7}{*}{ Dosha Karma } & Vataghna & - & - & - & - & - & - & - & - & - \\
\hline & Pittaghna & + & + & + & - & - & - & - & - & - \\
\hline & Kaphaghna & - & - & - & + & + & + & + & + & + \\
\hline & Tridoshaghna & - & - & - & - & - & - & - & - & - \\
\hline & Kapha-Vataghna & - & - & - & - & - & - & - & - & - \\
\hline & Kapha-Pittaghna & - & - & - & - & - & - & - & - & - \\
\hline & Vata-Pittaghna & - & - & - & - & - & - & - & - & - \\
\hline
\end{tabular}


References of Guna:

\begin{tabular}{|l|l|l|l|l|l|l|l|l|l|}
\hline Guna & C.S. & S.S. & $\begin{array}{l}\text { A.S./ } \\
\text { A.H. }\end{array}$ & B.N. & D.N. & M.N. & K.N. & R.N. & Sg. N. \\
\hline Gura & - & - & - & + & + & + & + & + & + \\
\hline Laghu & - & - & - & - & - & - & - & - & + \\
\hline Ushna & - & - & - & + & + & + & + & + & + \\
\hline Ruksha & - & - & - & - & - & - & - & - & - \\
\hline Snigdha & - & - & - & - & - & + & - & - & - \\
\hline
\end{tabular}

References of Karma:

\begin{tabular}{|c|c|c|c|c|c|c|c|c|c|c|}
\hline Karma & CS & $\mathrm{SS}$ & $\mathrm{AH}$ & $\mathrm{BN}$ & $\mathrm{DN}$ & $\mathrm{MN}$ & $\mathrm{KN}$ & $\mathrm{RN}$ & Sg.N & Sh.N \\
\hline Arshoghna & + & + & + & - & -8 & - & + & - & + & - \\
\hline Dahashamak & + & + & - & & - & - & - & - & - & - \\
\hline Jwarahar & + & + & $-\quad>1$ & - & - & - & - & + & + & - \\
\hline Kandughna & + & + & + & - & - & - & - & - & - & - \\
\hline Krumighna & + & - & - & - & - & + & - & - & + & - \\
\hline Kusthaghna & + & + & + & + & - & + & + & - & + & + \\
\hline Raktaprasadan & + & - & + & + & + & + & + & - & + & - \\
\hline Sandhaniya & + & + & - & - & - & - & - & - & - & - \\
\hline Shonitsthapan & + & - & + & + & - & + & - & - & + & - \\
\hline Shothahar & + & - & + & + & - & + & + & - & + & + \\
\hline Shulaghna & - & + & + & + & - & + & - & - & + & - \\
\hline
\end{tabular}




\begin{tabular}{|l|l|l|l|l|l|l|l|l|l|l|l|} 
Varnya & + & - & + & + & - & - & - & - & - & + \\
\hline Vishaghna & + & + & + & + & + & + & + & + & + & - \\
\hline Vrana-Ropan & + & + & + & + & + & + & + & - & + & - \\
\hline
\end{tabular}

\section{Discussion}

- Bhavprakash, Dhanvantari, Madanpal, Kaiyadev, Raj and Shaligram Nighantu have mentioned Ras of Majishtha-Madhur, Tikta, Kashaya; Virya - Ushna ; Vipak Madhur; Kaphaghna; Guru, Ushna Guna. Acharya Charak \& Vaghbhat mentioned - Kashaya rasatmak \& Pittaghna.

- Rasa - As Manjishtha being Madhur, Tikta and Kashaya rasatmak, its panchabhautik predominance is

Madhur - Prithavi + Jal

Tikta - Vayu + Akash

Kashaya - Vayu + Prithavi

- Virya - Ushna virya of Manjishtha indicates Agni mahabhuta predominance.

Vipak - Manjistha having Madhur vipak stated as per majority of the acharyas, has Prithavi and Jal mahabhut predominance.

- Guna - Manjistha possesses the following guna as per most of the reference texts which shows its mahabhut dominance is as follows-

- Guru-Prithavi + Jal

Ushna-Agni

\section{Conclusion}

Based on the above-mentioned analysis, it can be concluded that the Manjishtha is
Madhur, Tikta, Kashaya rasatmak; Ushna viryatmak; Madhur vipaki; Guru, Ushna, Snigdha gunatmak and Kapha-Pittahara.

Manjistha is Agni, Vayu and Prithvi mahabhut pradhan.

\section{References:}

1. Singh Prof. R.H. Charak Samhita of Agnivesh, Acharya Yadavaji Trikamji Vaidya ed.Varanasi, Chaukhamba Surbharati Prakashan,2017, p.no.32

2. Singh Prof. R.H. Charak Samhita of Agnivesh-Chikitasasthan, Acharya Yadavaji Trikamji Vaidya ed.Varanasi, Chaukhamba Surbharati Prakashan, 2017, p.no.456, 463, 473, $488,531$.

3. Chunekar Prof. Krushnachand Bhavaprakash Nigantu. Pandey Dr. G. S.Lateed.. Varanasi, Chaukhamba Bharati Acadamy, 2010.

4. Acharya P.V. Sharma; Kaiyadeva Nighantu; Chaukhambha Orientalia; 1979, p: 56

5. Vaidya, Shri Bapalaal Vaidya, Nigantu Aadarsh (Vol. 1). Varanasi: Chaukhamba Vishwabharati: Manjishthadi varga, ed. $1^{\text {st }}, 1968$, p.707709.

\section{Abbrevations}

- C. S. - Charak Samhita 
- S. S. - Sushrut Samhita

- A. H. - Ashtang Hridayam

- A.S. - Ashtang Samgrah

- D. N. - Dhanvantari Nigantu

- B. N. - Bhavaprakash Nigantu
- R.N. - Raj Nigantu

- M. N. - Madanpal Nigantu

- K. N. - Kaiyyadev Nigantu

- Sh. N. - Shodhal Nigantu

- Sg. N. - Shaligram Nigantu

Conflict of Interest: Non

Source of funding: Nil

Cite this article:

"A review article of Rasapanchak of Manjishtha (Rubia cordifolia Linn.) according to Ayurveda from Brihatrayi \& Various Nighantu."

Snehal Kumar Dabhole, Priyanka Dilip Chavan

Ayurlog: National Journal of Research in Ayurved Science- 2020; (8) (6):01- 05 